\title{
Original
}

\section{Estudio de la estacionalidad del dengue en la costa pacífica de Costa Rica (1999-2004)}

Roy Wong- McClure, Marilys Suárez- Pérez, Xiomara Badilla-Vargas

\section{Resumen}

El virus del dengue constituye la causa más común de enfermedades por arbovirus en el mundo. El estudio analiza el comportamiento estacional del dengue en la región Pacífico Central de Costa Rica, durante el período comprendido entre 1999 y 2004.

Materiales y métodos: La información fue tomada del Ministerio de Salud de Costa Rica y del Instituto Meteorológico Nacional. Para el análisis de la estacionalidad de dengue se utilizaron el número de casos de esta enfermedad para la estimación de promedios móviles y las características de los brotes epidémicos. Se definió como brote epidémico un total de 20 ó más casos de dengue por semana epidemiológica.

Resultados: Durante el período analizado se observó un incremento en el número de casos de dengue por año. Al observar la distribución de los casos incidentes del dengue cada año se resaltó un patrón anual estacional. Los brotes del dengue se presentaron al menos una vez al año, entre las semanas epidemiológicas 18-29 (abril-julio), que coincidían con la estación lluviosa.

Discusión: Se evidencia que en la región Pacífico Central, el dengue es una enfermedad predecible en cuanto a su comportamiento estacional, por lo que se recomienda intensificar las medidas de prevención para combatirla, así como preparar los servicios para la atención de pacientes, en las semanas epidemiológica previas a las de mayor promedio en el número de casos, según el patrón estacional.

Vigilancia Epidemiológica, Departamento de Salud Colectiva, Dirección de Desarrollo de Servicios de Salud, Caja Costarricense de Seguro Social

Abreviaturas: CCSS Caja Costarricense de Seguro Social; CDC, Centro para el control y prevención de las enfermedades de Estados Unidos de América; DE, Desviación Estándar; OPS, Organización Panamericana de la Salud

Correspondencia: Roy Wong rwong@ccss.sa.cr

Edificio Genaro Valverde. Piso II. Vigilancia Epidemiológica, Departamento de Salud Colectiva

ISSN 0001-6002/2007/49/1/36-39 Acta Médica Costarricense, (C2007 Colegio de Médicos y Cirujanos
Descriptores: Dengue, estacionalidad del dengue, dengue en Costa Rica

Key words: Dengue, Costa Rica

Recibido: 6 de junio de 2006

Aceptado: 17 de octubre de 2006

El virus del dengue es un virus ARN de la familia Flaviviridae, que constituye la causa más común de enfermedades por arbovirus en el mundo. La transmisión de la enfermedad se produce por medio de la picadura de su principal vector, el Aedes aegypti. ${ }^{1}$

La distribución del dengue se encuentra fundamentalmente en los trópicos ${ }^{2}$ y es el virus responsable de causar cada año, alrededor del mundo, un estimado de 50 a 100 millones de enfermos, incluyendo de 250.000 a 500.000 casos de dengue hemorrágico y cerca de 24.000 muertes. ${ }^{3,4} \mathrm{La}$ incidencia y el número de países considerados endémicos por dengue han incrementado en los últimos años. ${ }^{5}$

El dengue ha sido considerado la enfermedad viral transmitida por vectores más importante en el mundo para 2005, según el Centro para el Control y Prevención de las enfermedades de 
Estados Unidos de América (CDC), estimándose que alrededor de 2.5 billones de personas viven en áreas bajo riesgo de epidemias por dengue. ${ }^{6}$

En Costa Rica, durante 1993, se presentaron los primeros casos de dengue en Puntarenas y este se extendió posteriormente por todo el territorio nacional. Al igual que la tendencia en el resto del mundo, Costa Rica ha sufrido un incremento en la incidencia del dengue, presentando varias epidemias en los años siguientes, con la presencia de algunos casos de dengue hemorrágico. ${ }^{7,8}$

Las epidemias de dengue causan gran impacto socioeconómico, que se refleja al tomar en cuenta todos los programas de erradicación del vector producto de los brotes repetitivos, los días laborales perdidos, la atención y seguimiento de los pacientes diagnosticados con esta enfermedad, las hospitalizaciones y, lo más importante, la vida de las personas. ${ }^{9-11}$

El costo de la atención del dengue ha evidenciado un incremento importante en los últimos años, debido al aumento en el número de casos incidentes de la enfermedad detectados en todo el país.

Por lo tanto, el dengue se ha convertido en una gran preocupación para la salud pública de Costa Rica; según cifras brindadas por la Caja Costarricense de Seguro Social (CCSS) para 2002 el costo generado por las consultas de dengue fue de $\$ 125.264 .413$, la estancia hospitalaria de $\$ 332.690 .380$ y las incapacidades, $\$ 7.921 .649$. Estas cifras produjeron un costo total en atención del dengue de 465.876.441 colones. ${ }^{12}$

Se ha descrito que el dengue presenta un patrón estacional en donde se haya un incremento de la incidencia durante la estación lluviosa. ${ }^{13,14}$ Desde hace varios años el dengue forma parte del perfil epidemiológico del la región Pacífico Central, una de las que ha reportado mayor cantidad de casos por años, de modo que el estudio analizará el comportamiento estacional del dengue en la costa Pacífica de Costa Rica durante el período comprendido entre 1999 y 2004 y su relación con la precipitación pluvial.

\section{Materiales y métodos}

\section{Fuente de información}

Se utilizó como fuente de información la notificación oficial de casos de dengue por semana epidemiológica brindada por el Ministerio de Salud, durante el período comprendido entre 1999 y 2004, en la en la región Pacífico Central. Un caso de dengue es definido para la notificación oficial como todo aquel sospechoso por esta enfermedad, corroborado por las pruebas inmunológicas del laboratorio de referencia. En caso de epidemia, el paciente sospechoso que resida en esa área. ${ }^{8}$
Los registros de precipitaciones se recopilaron en el Instituto Meteorológico Nacional (IMN), con datos históricos hasta años recientes. Estos fueron registrados en diferentes estaciones de la región Pacífico Central, entre ellas la de Puntarenas, Punta Leona, Jacó, Parrita y Quepos.

\section{Procesamiento de datos y análisis de la información}

Para el análisis de la información se realizó en la primera etapa la estimación de las medidas de tendencia central y de dispersión para el número de casos reportados por año. Posteriormente se determinó la estacionalidad por medio de la estimación de medias móviles, con un rango establecido de 3 semanas.

Luego se efectuó la estimación de las semanas donde se evidenciaron las características de las diferentes brotes, tales como la semana del incremento en el número promedio de casos, su pico máximo, la semana de descenso y como la duración de los brotes epidémicos.

Para fines de la investigación, se definió brote epidémico por dengue cuando se contabilizaron un total de 20 ó más casos reportados por semana epidemiológica.

Para el procesamiento de la información se utilizó en la primera etapa el software estadístico Epi Info 3.3.2 (CDC2005) y para la determinación de las medias móviles, el Minitab versión 12.2, contemplando número de casos por semana epidemiológica y años de ocurrencia de estos.

\section{Mediciones meteorológicas}

La determinación de la precipitación pluvial promedio se calculó con base en los datos del Instituto Meteorológico Nacional en sus estaciones de Puntarenas, Punta Leona, Jacó, Parrita y Quepos. Las mediciones fueron realizadas con pluviómetros tipo Hellmann ${ }^{15,16}$ y se obtuvo un promedio mensual con las determinaciones de por lo menos 5 años de datos recopilados.

\section{Resultados}

Durante el período de observación se contabilizaron, para la región Pacífico Central, 10308 casos notificados de dengue, tanto en su manifestación clásica como hemorrágica.

Para cada uno de los años en estudio se evidenció que el año con mayor promedio semanal de casos fue 2003, con 43.0 casos (DE: 40.3), seguido por 2004, con 39.5 casos (DE: 23.7) y 38.1 casos (DE: 39.3) para 2000 (Cuadro 1).

En los casos oficiales reportados de dengue durante la observación, se evidenció un comportamiento creciente entre 1999 y el 2004, con un descenso en el número de casos en 2001 y 2002, y de menor magnitud en 2004 (Figura 1). 


\begin{tabular}{|ccc|}
\hline $\begin{array}{c}\text { Cuadro 1. Promedio semanal de casos de } \\
\text { dengue notificados, según año en estudio. } \\
\text { Región Pacífico Central. }\end{array}$ \\
\hline Año & Promedio & D.E \\
\hline 1999 & 20.8 & 28.8 \\
2000 & 38.1 & 39.3 \\
2001 & 25.0 & 24.2 \\
2002 & 30.9 & 30.6 \\
2003 & 43.0 & 40.3 \\
2004 & 39.5 & 23.7 \\
\hline \multicolumn{2}{l}{ Fuente: Ministerio de Salud } \\
\hline
\end{tabular}

Al estudiar la estacionalidad de los casos notificados según semana epidemiológica de fecha de inicio de síntomas, se evidenció que el incremento de los casos incidentes de dengue se produjo entre la semana 15 y la 26 (abril - junio), siendo la 15 (abril) la que refirió con mayor frecuencia el incremento en el número de casos, en dos de los años en observación. La presencia estacional del número de casos incidentes de dengue evidenció estar asociada con el incremento local en la precipitación pluvial (Figura 1).

Con respecto a la estacionalidad y la presencia del pico máximo de casos notificados, los años de 1999 a 2001 contaron con la presencia del mayor número de casos incidentes en la semana 34 (agosto), y para los años entre 2002 y 2004, la condición se encontró entre la semana 30 y la 39 (julio-septiembre; Cuadro 2).

$\mathrm{Al}$ estudiar la diferencia entre el punto de ascenso y el descenso de las medias móviles calculadas, para determinar la duración de los brotes epidémicos, se evidenció que el promedio de duración de las diferentes epidemias fue de 25 semanas, con un rango de entre 19 y 28.

Al evaluar entre la semana de inicio del brote epidémico y el pico máximo, como medida de la duración en el incremento del número de casos, se presentó un promedio de 11 semanas, con un rango de 5 a 18 (Cuadro 2).

\section{Discusión}

En el estudio se analizó la distribución de los casos notificados por dengue, en la región Pacífico Central de Costa Rica, en el período comprendido entre 1999 y 2004. Se evidenciaron similitudes al tomar en cuenta la semana epidemiológica cuando inicia el brote del dengue, la semana cuando se contabilizó el mayor número de casos, la duración del brote y la semana cuando finaliza este.

Al observar la distribución de los casos incidentes de dengue cada año se resaltó un patrón anual estacional. Se estableció que al superar 20 casos reportados entre las semanas epidemiológicas 18 y 29 (abril-julio), se

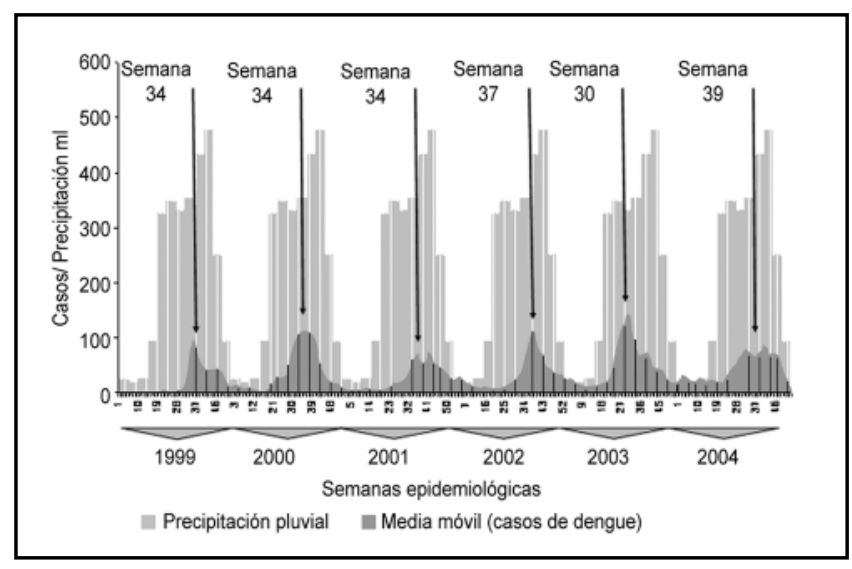

Figura 1. Medias móviles de casos notificados de dengue, según promedios mensuales de precipitación pluvial. Costa pacífica de Costa Rica, 1999-2004.

desencadena un comportamiento creciente que llega a un pico máximo en un promedio de 11 semanas, para luego descender y resolverse el brote en un tiempo promedio de 14 semanas posteriores al pico máximo. Se observó que el brote epidémico por dengue se presentaba al menos una vez al año, en un período de aparición que coincidió con la estación lluviosa de la región estudiada.

La estación lluviosa de la región Pacífico Central se extiende generalmente desde mayo hasta octubre, ${ }^{16}$ período cuando se presentaron los brotes de dengue. Esta tendencia se ha observado también en Brasil, Puerto Rico y otros países cuya incidencia de casos por dengue incrementa durante la época lluviosa.., 13,14

Se ha documentado que para que el Aedes aegypti se reproduzca, es necesario su acceso a aguas limpias y estancadas para el depósito de los huevos. El incremento de estos sitios, debido a una mayor cantidad de desechos expuestos a la intemperie y a la ineficiencia en su recolección y manejo, ha provocado un incremento en el número de vectores. Por lo tanto, la estación lluviosa se encuentra estrechamente relacionada con la proliferación del mosquito, al multiplicarse el número de criaderos.

\section{Cuadro 2. Semanas epidemiológicas cuando inicia el brote epidémico, su pico máximo, fin y duración. Pacífico Central, 1999-2004}

\begin{tabular}{|ccccc|}
\hline Año & $\begin{array}{c}\text { Inicio de } \\
\text { brote }\end{array}$ & $\begin{array}{c}\text { Pico } \\
\text { máximo }\end{array}$ & $\begin{array}{c}\text { Fin de } \\
\text { brote }\end{array}$ & $\begin{array}{c}\text { Duración } \\
\text { del brote }\end{array}$ \\
\hline 1999 & 29 & 34 & 48 & 19 \\
2000 & 18 & 34 & 45 & 27 \\
2001 & 26 & 34 & 50 & 24 \\
2002 & 27 & 37 & 51 & 24 \\
2003 & 19 & 30 & 47 & 28 \\
2004 & 21 & 39 & 49 & 28 \\
\hline Promedio & 23 & 35 & 48 & 25 \\
general & & & & \\
\hline Fuente: Ministerio de Salud, 2005 & & \\
\hline
\end{tabular}


Por la relación existente entre la precipitación pluvial y el conocido patrón estacional de la incidencia de la enfermedad, ${ }^{11,12}$ esta puede verse afectada por cambios climatológicos globales.

En conclusión, se puede determinar que el dengue en el pacífico de Costa Rica es una enfermedad predecible, con estacionalidad anual definida y muy vinculada con la precipitación pluvial registrada.

Es importante recomendar el fortalecimiento de las medidas de prevención, así como preparar los servicios de salud para prevenir y atender a los pacientes con dengue en el momento cuando el patrón estacional indica el inicio de su reaparición lo mismo que identificar el rango de intensificación de las acciones durante las semanas epidemiológicas entre la 18 y 29 (abril-julio), la cuales corresponden a las medias de duración de la epidemias.

A pesar de la necesidad de intensificar las medidas de control en las semanas establecidas, es preciso mantener la continuidad de la educación a la población en todas las épocas del año.

También se recomienda que los sistemas de vigilancia epidemiológica y alerta de los casos de dengue trabajen en coordinación con los Institutos Meteorológicos, en procura de una mayor precisión al intensificarse las medidas de prevención y control.

De tener éxito en las campañas de prevención del dengue, se lograrían brotes de menor duración y magnitud, lo que reduciría el impacto social y económico en los pueblos.

\section{Agradecimiento:}

A los funcionarios del Instituto Meteorológico Nacional, por brindar datos de la precipitación pluvial en la Región Pacífico Central.

\section{Abstract}

Aim: This study analyzed the Dengue's seasonal behaviour in the Central Pacific Region of Costa Rica, during the period from 1999 to 2004. It is known that the dengue virus is the most common cause of arbovirus diseases in the world.

Material and methods: We got the data from the Costa Rican Ministry of Health and the National Meteorological Institute. From this data, moving averages were calculated to evaluate its seasonal behaviour and the features of the outbreaks. In this study an outbreak of dengue is defined when there are 20 or more cases reported per week.

Results: During this period under study, an increase in the number of dengue cases per year was observed; its numerical behaviour throughout this interval, reflected a pattern in which the outbreaks appeared between the $18^{\text {th }}$ through the $29^{\text {th }}$ epidemiological week (April - July) which coincided with the rainy season in the region.

Discusion: The conclusion drawn from this study is that in the Central Pacific region, the dengue disease shows a predictable pattern in relation to the rainy season. For this reason, intensifying preventive measures, as well as, preparedness for patient treatment is strongly recommended, before the epidemiological week with highest average of cases and the seasonal pattern.

\section{Referencias}

1. Organización Panamericana de Salud. El control de las enfermedades transmisibles. 17ª ed. Washington, DC: OPS; 2001.

2. Fauci A, Braunwald E, Martin J, Kasper D, Isselbacher K, Wilson J et al. Principios de medicina interna. 14ª ed. Nueva York: Mc Graw Hill; 1998.

3. Gibbons R., Vaughn W. Dengue: An Escalating Problem. Clinical Review. BMJ 2002; 321: 1563-1566.

4. Gubler D. Dengue and Dengue Hemorrhagic Fever. Clin Microbiol. Rev. 1998; 11:480-96.

5. Organización Mundial de la Salud. Dengue and Dengue Haemorrhagic Fever. Revisado el 4 de marzo 2006 Disponible en: http://www.who. int/mediacentre/factsheets/fs117/en/

6. Centres for Disease Control and Prevention. Dengue Fever. Revisado el 5 de marzo 2006. Disponible en: http://www.cdc.gov/ncidod/dvbid/ dengue/

7. Alfaro A, Navas L, Pérez E, Barrantes J, Rodríguez M, Pizarro D et al. Hallazgos clínicos detectados al ingreso de los pacientes hospitalizados para predecir la evolución a dengue hemorrágico. Memorias de Academia Nacional de Ciencias. El Dengue 2001;7:49-66.

8. Ministerio de Salud, Comisión Técnica Interinstitucional de Dengue. Normas técnicas para el control del dengue y dengue hemorrágico. $1^{\mathrm{a}}$ ed. San José: Ministerio de Salud; 2000.

9. Von S, López R, Woodall J, Morens D, Chiribiga J, Casta A. Epidemic Dengue Fever in Puerto Rico, 1977: A Cost Análisis. Am J Trop Med Hyg 1979;28:1040-4

10. OPS. Hemispheric plan to expand and intensify efforts to combat Aedes aegypt. Revisado el 7 marzo 2006. Disponible en: www.paho. org/English/AD/DPC/CD/doc406.pdf

11. Organización Panamericana de la Salud. Dengue y dengue hemorrágico en las Américas: guías para su prevención y control. Publicación Científica no. 548. 1995. Washington, D.C; OPS, 1995.

12. C.C.S.S. Costo de la atención del dengue. Revisado el 25 de mayo 2006. Disponible en: http://www.ccss.sa.cr/geradm/dircom/elseguro/ dengue/inversion_total.html

13. Siqueira J, Turchi C, Coelho G, da Rocha A. Dengue and dengue hemorrhagic fever, Brazil, 1981-2002. Emer Infec Dis. 2005;11:48-53

14. Perez R. Dengue surveillance, United States, 1986- 1992. MMWR CDC Surveill Sum. 1994 July 22;43:7-19.

15. Comité Regional de Recursos Hidráulicos. Observaciones meteorológicas. Tegucigalpa: CETTNA; 1977.

16. Instituto Meteorológico Nacional. El clima en Costa Rica. Revisado el 27 de julio 2006. Disponible en: http://www.imn.ac.cr/educa/clima/ clima\%20en\%20costa\%20rica.htm 